\title{
The efficacy of different doses of ulinastatin in the treatment of severe acute pancreatitis
}

\author{
Hai Wang He, Hong Zhang \\ Department of Emergency Medicine, The First Affiliated Hospital of Anhui Medical University, Hefei 230022, China \\ Contributions: (I) Conception and design: H Zhang; (II) Administrative support: H Zhang; (III) Provision of study materials or patients: H Zhang; (IV) \\ Collection and assembly of data: HW He; (V) Data analysis and interpretation: HW He; (VI) Manuscript writing: All authors; (VII) Final approval of \\ manuscript: All authors. \\ Correspondence to: Hong Zhang, MD. Department of Emergency Medicine, The First Affiliated Hospital of Anhui Medical University, 218 Jixi Road, \\ Hefei 230022, China. Email: zhanghong20070703@163.com.
}

\begin{abstract}
Background: Severe acute pancreatitis (SAP) is a severe form of inflammatory disease with a high mortality rate. Ulinastatin, a urinary trypsin inhibitor, has anti-inflammatory properties and may be beneficial to critically ill patients with SAP. Nevertheless, there is currently insufficient evidence to conclude whether there is a dose-effect relationship between ulinastatin and SAP treatment outcomes. The present study examined the efficacy of ulinastatin at different doses in the treatment of SAP.

Methods: A retrospective study was conducted examining the clinical outcomes of 130 SAP patients. Patients were categorized into a control group and three groups receiving different daily doses of ulinastatin (200,000; 400,000; and 600,000 IU). The study compared the 1-week mortality rate; the Acute Physiology and Chronic Health Evaluation II (APACHE-II) score; abdominal pain relief time; time to recover a normal heart and respiratory rate; blood amylase, glucose, C-reactive protein, and procalcitonin levels; and white blood cell (WBC) count among the different groups.
\end{abstract}

Results: The 400,000 and 600,000 IU groups had significantly lower mortality rates and WBC count compared to the 200,000 IU group $(\mathrm{P}<0.05)$. Furthermore, the 400,000 IU group had a significantly shorter abdominal pain relief time compared to the 200,000 IU group $(\mathrm{P}<0.05)$. Compared to the 200,000 IU group, the 600,000 IU group had significantly shorter time to recover a normal respiratory rate and a lower APACHE-II score $(\mathrm{P}<0.05)$.

Conclusions: Ulinastatin can improve the clinical outcomes of patients with SAP but efficacy varies with the dosage.

Keywords: Severe acute pancreatitis (SAP); mortality; ulinastatin; systemic inflammatory response syndrome; multiple organ deficiency syndrome

Submitted Aug 18, 2019. Accepted for publication Feb 14, 2020.

doi: 10.21037/apm.2020.04.19

View this article at: http://dx.doi.org/10.21037/apm.2020.04.19

\section{Introduction}

Acute pancreatitis (AP) is one of the most common diseases of the gastrointestinal tract and carries a significant emotional, physical, and financial burden $(1,2)$. AP is characterized by pancreatic self-digestion, in which pancreatic enzymes cause injury to the pancreas and lead to glandular dysfunction, as well as repercussions in remote organs and systems (3).
The global pooled incidence of AP is 34 cases per 100,000 people per year, equally affecting men and women but predominantly middle-aged or older individuals (4).

In the United States, there are approximately 300,000 patients admitted to hospitals each year, resulting in medical expenses of USD 2.64 billion (1). The 2012 revised Atlanta classification stratified AP into mild, moderately severe, and severe acute pancreatitis (SAP) based on the presence of 
persistent organ failure and complications (5). Of these, SAP is a common critical illness seen in the clinical emergency setting, characterized by its fast onset and progression and high mortality rate. If organ failure cannot be resolved within $48 \mathrm{~h}$, SAP will develop into single organ failure which may persist to the late phase of $\mathrm{AP}$ and even affect other organs. The lung is the most commonly affected extra-pancreatic organ in $\mathrm{AP}$ and this is frequently followed by acute kidney and cardiovascular injury (6). Current treatments for SAP are limited to conservative medical management (7); however, there is no medication with confirmed efficacy. In China, protease inhibitors are widely used for the treatment of AP and demonstrate positive treatment effects (8). Ulinastatin is a glycoprotein which can be obtained from human urine or produced synthetically. Proteolytic enzymes, including trypsin, elastase, and plasmin can be effectively inhibited by ulinastatin, lipase, and amylase, thus relieving the systemic inflammatory response and diminishing the severity of pancreatitis (9-12). However, the dose-response effect of ulinastatin in SAP has not been established. This study explored the effect of different ulinastatin doses for SAP treatment.

\section{Methods}

\section{Setting and subjects}

This retrospective study was conducted in the internal medicine emergency department and intensive care unit of the First Affiliated Hospital, Anhui Medical University, Hefei, China, from January 2013 to May 2018. All patients fulfilled the diagnostic criteria for SAP and were screened for potential eligibility. According to the Atlanta classification, SAP is characterized by persistent organ failure, including single or multiple organ failure. Patients were included if they were aged $\geq 18$ years and had been receiving ulinastatin for over 1 week. Patients were excluded if they had preexisting severe cardiovascular, pulmonary, hepatic, or renal dysfunction before SAP onset; if there was an incomplete set of clinical data; if there was a comorbid malignant tumor; or if there was any underlying disease. Patients were distributed into a control group $(\mathrm{n}=26)$, a 200,000 IU group $(\mathrm{n}=21)$, a 400,000 IU group $(n=56)$, and a 600,000 IU group $(n=27)$ based on their total daily dose of ulinastatin.

\section{Ethics statement}

The study was approved by the ethics committee of the
First Affiliated Hospital, Anhui Medical University (No. Quick-PJ2019-17-21). Informed consent was waived because the study was retrospective in design. The study was performed in accordance to the Helsinki Declaration. Individual patient data were anonymized and stored in an encrypted computer.

\section{Treatment}

Patients in the control group received a comprehensive range of treatment including fasting, gastrointestinal decompression, early aggressive fluid resuscitation, spasmolysis, purgation, acid suppression, somatostatins (Stilamin $6 \mathrm{mg} /$ day with continuous intravenous pump infusion) to suppress pancreatin secretion, external application of mirabilite, and enteral nutrition. Patients with biliary pancreatitis were treated with antibiotics, while those with biliary obstruction received timely endoscopic obstruction removal. Patients with pancreatitis secondary to hyperlipidemia with triglyceride levels $>11.3 \mathrm{mmol} / \mathrm{L}$ were treated by plasmapheresis and patients with Multiple Organ Dysfunction Syndrome (MODS) received support therapy specific to the failing organs. The 200,000, 400,000, and $600,000 \mathrm{IU}$ treatment groups received their respective ulinastatin doses intravenously for at least 7 days in addition to the control group treatments. The use of different doses of ulinastatin (Guangdong Techpool Bio-pharma Co. Ltd., Guangdong, China) was determined by the attending physician and not all patients with SAP received ulinastatin. Data about the use of ulinastatin were extracted from electronic healthcare records. Ulinastatin was discontinued if the general condition of the patient improved or if allergy was suspected.

\section{Clinical variables}

A range of laboratory variables were obtained on day 1 after admission, including white blood cell (WBC) count and procalcitonin (PCT), C-reactive protein (CRP), blood glucose, and blood amylase levels. If more than one measurement was obtained, the maximum value was recorded. These indices were also obtained on day 7 and the difference between the days 1 and 7 values was then calculated. The Acute Physiology and Chronic Health Evaluation II (APACHE-II) score was calculated by using the clinical and laboratory variables within 24 hours after admission. The APACHE-II was also calculated on the $7^{\text {th }}$ day after admission and the values with the maximum 


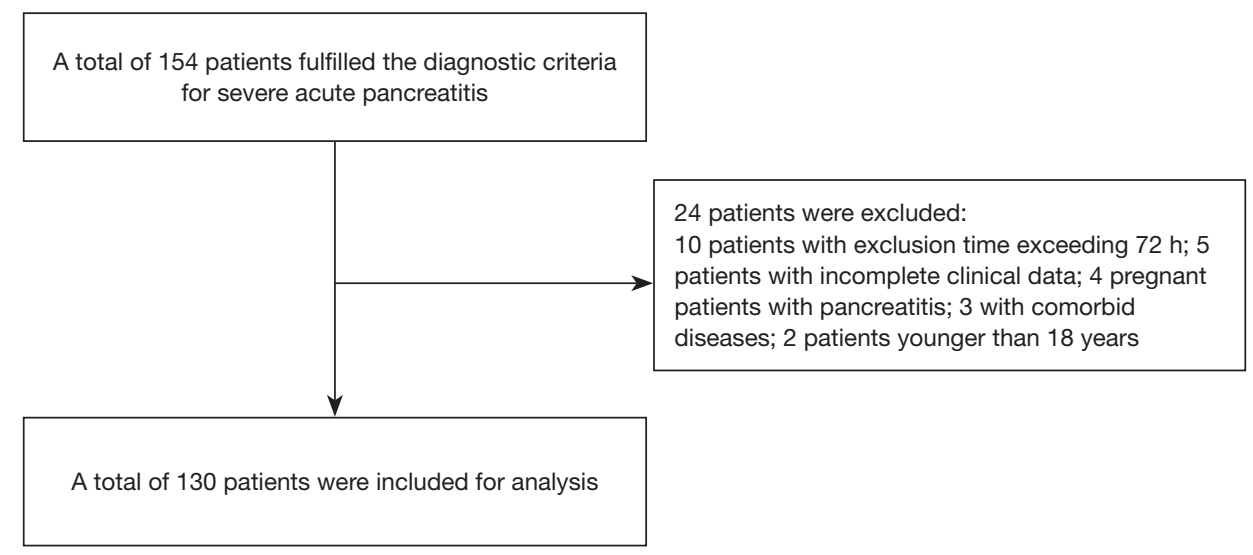

Figure 1 Flowchart showing the patient selection protocol.

point score were used. The time taken to recover a normal heart and respiratory rate, abdominal distention relief time, and the previously mentioned parameters were recorded according to the actual situation of each patient.

\section{Outcomes}

Mortality was defined as the vital status at 7 days after emergency department admission. In this study, efficacy was classified into 4 levels: cure, improvement, no improvement, and death. Cure refers to the elimination of abdominal signs and symptoms and the normalization of laboratory values within 1 week of treatment. Improvement refers to lessening clinical signs and symptoms and improving laboratory values within 1 week of treatment. No improvement refers to either a lack of significant change or worsening in clinical signs and symptoms and laboratory values without mortality after 1 week of treatment. Death refers to the worsening of the disease leading to mortality after 1 week of treatment. The calculation used for the 1-week mortality rate was as follows: (number of deaths/total number of patients in each group) $\times 100 \%$. Other outcomes included APACHEII scores, the time to recover a normal heart and respiratory rate, abdominal distention relief time, abdominal pain relief time, side effects, and time taken to recover normal blood levels of endotoxin, CRP, PCT, and WBC.

\section{Statistical analysis}

All statistical analyses were conducted using SPSS version 22.0 (IBM Corp., Armonk, NY, USA). Quantitative data are reported as mean \pm standard deviation. Categorical data are reported as percentages. The $\chi^{2}$ test or analysis of variance (ANOVA) were used to compare data between groups. $\mathrm{P}<0.05$ was considered to indicate statistical significance.

\section{Results}

\section{Basic patient data and characteristics}

Initially, a review of medical charts identified a total of 154 patients who fulfilled the diagnostic criteria for SAP. In-depth screening excluded 24 of these patients; 10 were excluded because admission time exceeded 72 hours, 5 were excluded due to incomplete clinical data, 4 were excluded because they were pregnant females with pancreatitis, 3 were excluded because they had comorbid diseases, and 2 were excluded because they were under 18 years old. Consequently, a total of 130 patients were included in the final analysis (Figure 1).

Of the 130 patients with SAP included in this study, the control group consisted of 13 male and 13 female patients with a mean age of $46.31 \pm 14.70$ years (range, $21-79$ years); the 200,000 IU group consisted of 11 male and 10 female patients with a mean age of $43.29 \pm 10.59$ years (range, $21-$ 66 years); the 400,000 IU group consisted of 26 male and 30 female patients with a mean age of $44.48 \pm 15.14$ years (range, $19-80$ years) and the 600,000 IU group consisted of 13 male and 14 female patients with a mean age of $45.63 \pm 14.36$ years (range, 24-67 years). The four most common causes of SAP were biliary causes, hyperlipidemia, alcohol, and other factors. There were no statistically significant differences in age, gender, disease etiology, and basic demographics among patients in different groups $(\mathrm{P}>0.05$; Table 1). 
Table 1 Comparisons of age, sex, and etiology among patients in different groups

\begin{tabular}{lcccc}
\hline Group & N & Mean age (years) & Sex (n, male/female) & Etiology (n) \\
\hline Control group & 26 & $46.31 \pm 14.70$ & $13 / 13$ & $15 / 5 / 4 / 2$ \\
200,000 IU group & 21 & $43.29 \pm 10.59$ & $11 / 10$ & $12 / 4 / 4 / 1$ \\
400,000 IU group & 56 & $44.48 \pm 15.14$ & $26 / 30$ & $37 / 9 / 6 / 4$ \\
600,000 IU Group & 27 & $45.63 \pm 14.36$ & $13 / 14$ & $17 / 5 / 3 / 2$ \\
F/ $\chi^{2}$ value & & 0.214 & 0.248 & 1.632 \\
P value & & 0.887 & 0.970 & 0.996
\end{tabular}

F, Fisher's exact test; $\chi^{2}$, chi-squared test.

Table 2 Comparison of 1 -week mortality rates among patients in different groups (n, \%)

\begin{tabular}{|c|c|c|c|c|c|c|}
\hline Group & $\mathrm{N}$ & Cure & Improvement & No improvement & Death & Mortality rate (\%) \\
\hline 200,000 IU group & 21 & 2 & 11 & 1 & 7 & 33.33 \\
\hline 400,000 IU group & 56 & 4 & 44 & 5 & 3 & $5.36^{a, b}$ \\
\hline 600,000 IU group & 27 & 2 & 20 & 3 & 2 & $7.41^{\mathrm{b}}$ \\
\hline$P$ value & - & - & - & - & & 0.003 \\
\hline
\end{tabular}

${ }^{a}$, vs. control group, $\mathrm{P}<0.05$; $^{\mathrm{b}}$, vs. 200,000 IU group, $\mathrm{P}<0.05$.

\section{Comparison of mortality rates among different groups}

The mortality rate was $26.92 \%, 33.33 \%, 5.36 \%$, and $7.41 \%$ in the control, 200,000, 400,000, and 600,000 IU groups, respectively. The mortality rate in the 400,000 IU group was lower than that of the control group $(\mathrm{P}<0.05)$. The mortality rates of the 400,000 and 600,000 IU groups were lower than that of the 200,000 IU group $(\mathrm{P}<0.05$; Table 2$)$.

\section{Comparison of APACHE-II scores among different groups at admission and after one week of treatment}

The difference between the APACHE-II scores of the treatment groups and the control group at admission and at 1 week was statistically significant $(\mathrm{P}<0.05)$. The difference between the APACHE-II score of the 200,000 IU group and the $600,000 \mathrm{IU}$ group was also statistically significant $(\mathrm{P}<0.05$; Table 3).

\section{Comparison of clinical indicators among different groups}

Statistically significant differences were found in clinical indicators (abdominal pain relief time, time to recover a normal heart and respiratory rate) between the 400,000 and 600,000 IU groups and the control group $(\mathrm{P}<0.05)$. Moreover, the 400,000 IU group had a shorter abdominal pain relief time compared to the $200,000 \mathrm{IU}$ group $(\mathrm{P}<0.05)$. The time to recover a normal respiratory rate was shorter in the 600,000 IU group than in the 200,000 IU group $(\mathrm{P}<0.05)$. However, the time to recover a normal heart rate was not statistically different among groups with different ulinastatin doses $(\mathrm{P}>0.05$; Table 4).

\section{Comparison of differences in serological indicators at admission and 1 week after treatment among patients in different groups}

There were significant differences in blood glucose and CRP levels and WBC count between the ulinastatin treatment groups and the control group $(\mathrm{P}<0.05)$. However, there was no significant difference in blood glucose and CRP levels among the treatment groups $(\mathrm{P}>0.05)$. The WBC count of the 600,000 IU group was significantly different from that of the 200,000 and 400,000 IU groups $(\mathrm{P}<0.05)$. There was no significant difference in blood 
Table 3 Comparison of APACHE-II scores among patients in different groups at admission and after treatment for 1 week

\begin{tabular}{lcccc}
\hline \multirow{2}{*}{ Group } & $\mathrm{N}$ & \multicolumn{2}{c}{ APACHE-II score } & \multicolumn{2}{c}{$\begin{array}{c}\text { Difference between APACHE-II } \\
\text { scores at admission and 1 week }\end{array}$} \\
\cline { 3 - 5 } & & At admission & 1 week & $2.31 \pm 1.49$ \\
Control group & 26 & $17.85 \pm 2.89$ & $15.54 \pm 2.06$ & $3.57 \pm 1.66^{\mathrm{a}}$ \\
200,000 IU group & 21 & $16.76 \pm 2.68$ & $13.19 \pm 1.99$ & $4.11 \pm 1.63^{\mathrm{a}}$ \\
400,000 IU group & 56 & $16.21 \pm 1.72$ & $12.11 \pm 1.74$ & $4.62 \pm 1.52^{\mathrm{a}, \mathrm{b}}$ \\
600,000 IU group & 27 & $17.07 \pm 2.22$ & $12.44 \pm 1.97$ & 10.97 \\
F value & - & - & - & 0.000 \\
P value & - & - & - & 0.07 \\
\hline
\end{tabular}

Data are presented as mean \pm standard deviation. ${ }^{\text {a }}$, vs. control group, $\mathrm{P}<0.05$; $^{\mathrm{b}}$, vs. 200,000 IU group, $\mathrm{P}<0.05$.

Table 4 Comparison of clinical parameters among patients in different groups

\begin{tabular}{lccc}
\hline Group & Abdominal pain relief time (days) & Time to recover a normal respiratory rate (days) & Time to recover a normal heart rate (days) \\
\hline Control group & $9.65 \pm 3.05$ & $10.85 \pm 3.25$ & $11.08 \pm 2.94$ \\
200,000 IU group & $8.91 \pm 2.07$ & $9.95 \pm 1.77$ & $10.57 \pm 2.54$ \\
400,000 IU group & $7.32 \pm 2.64^{\mathrm{a}, \mathrm{b}}$ & $8.98 \pm 2.39^{\mathrm{a}}$ & $9.34 \pm 2.79^{\mathrm{a}}$ \\
600,000 IU group & $7.67 \pm 1.98^{\mathrm{a}}$ & $8.00 \pm 2.51^{\mathrm{a}, \mathrm{b}}$ & $9.07 \pm 2.66^{\mathrm{a}}$ \\
F value & 6.03 & 6.38 & 3.55 \\
P value & 0.001 & 0.000 & 0.016 \\
\hline
\end{tabular}

Data are presented as mean \pm standard deviation. ${ }^{a}$, vs. control group, $\mathrm{P}<0.05$; $^{\mathrm{b}}$, vs. 200,000 IU group, $\mathrm{P}<0.05$.

Table 5 Comparison of differences in serological indicators at admission and 1 week after treatment among patients in different groups

\begin{tabular}{|c|c|c|c|c|c|}
\hline Group & $\begin{array}{l}\text { Blood amylase difference } \\
\qquad(\mathrm{U} / \mathrm{L})\end{array}$ & $\begin{array}{l}\text { Blood glucose difference } \\
\qquad(\mathrm{mmol} / \mathrm{L})\end{array}$ & $\begin{array}{l}\text { CRP difference } \\
\qquad(\mathrm{mg} / \mathrm{L})\end{array}$ & $\begin{array}{l}\text { PCT difference } \\
(\mathrm{ng} / \mathrm{mL})\end{array}$ & $\begin{array}{l}\text { WBC count difference } \\
\qquad\left(10^{12} / L\right)\end{array}$ \\
\hline Control group & $119.57 \pm 227.06$ & $1.07 \pm 3.46$ & $40.76 \pm 26.03$ & $1.10 \pm 1.80$ & $0.75 \pm 4.78$ \\
\hline 400,000 IU group & $164.64 \pm 271.25$ & $3.11 \pm 3.50^{\mathrm{a}}$ & $74.21 \pm 34.89^{a}$ & $0.94 \pm 2.85$ & $5.71 \pm 5.13^{\mathrm{a}, \mathrm{b}}$ \\
\hline 600,000 IU group & $219.03 \pm 130.54$ & $4.30 \pm 2.93^{\mathrm{a}}$ & $80.72 \pm 21.28^{a}$ & $1.42 \pm 2.56$ & $10.03 \pm 6.88^{\mathrm{a}, \mathrm{b}}$ \\
\hline$P$ value & 0.339 & 0.005 & 0.000 & 0.835 & 0.000 \\
\hline
\end{tabular}

Data are presented as mean \pm standard deviation. ${ }^{a}$, vs. control group, $\mathrm{P}<0.05 ;{ }^{b}$, vs. 200,000 IU group, $\mathrm{P}<0.05$. CRP, C-reactive protein; $\mathrm{PCT}$, procalcitonin; WBC, white blood cell.

amylase and PCT levels at 1 week when compared across patients in different groups $(\mathrm{P}>0.05$; Table 5$)$.

\section{Multi-factor logistic regression analysis}

Logistic regression analysis was carried out using patient death as the dependent variable and APACHE-II score; abdominal pain relief; time to recover a normal heart and respiratory rate; blood amylase, blood glucose, CRP, and PCT levels; and WBC count differences as independent variables. The results showed that the time to recover a normal heart rate, blood amylase levels, and APACHE-II score differences should be considered as risk factors for SAP-related mortality (Table 6). 
Table 6 Multivariate logistic regression analysis of factors affecting the prognosis of patients with SAP

\begin{tabular}{|c|c|c|c|c|c|c|}
\hline Risk factor & $\beta$ & SE & Wald & $\mathrm{P}$ & OR & $95 \% \mathrm{Cl}$ \\
\hline Time to recover a normal respiratory rate & 0.446 & 0.300 & 2.219 & 0.136 & 1.563 & $0.869-2.812$ \\
\hline Time to recover a normal heart rate & -0.782 & 0.290 & 7.287 & 0.007 & 0.458 & $0.259-0.807$ \\
\hline Blood amylase difference & -0.003 & 0.001 & 4.879 & 0.027 & 0.997 & $0.994-1.000$ \\
\hline CRP difference & 0.015 & 0.010 & 2.242 & 0.134 & 1.015 & $0.995-1.036$ \\
\hline PCT difference & 0.083 & 0.136 & 0.374 & 0.541 & 1.087 & $0.832-1.420$ \\
\hline WBC count difference & 0.120 & 0.065 & 3.397 & 0.065 & 1.127 & $0.992-1.280$ \\
\hline APACHE-II score difference & 0.654 & 0.218 & 9.013 & 0.003 & 1.923 & $1.255-2.947$ \\
\hline
\end{tabular}

SAP, severe acute pancreatitis; SE, standard error; OR, odds ratio; Cl, confidence interval; CRP, C-reactive protein; PCT, procalcitonin; WBC, white blood cell; APACHE-II, Acute Physiology and Chronic Health Evaluation II.

\section{Discussion}

Previous research has shown that systemic inflammation reaction syndrome can occur even in the early stages of SAP (13-15), while MODS can occur in severe cases (8). The mechanism underlying the pathogenesis of SAP is commonly believed to involve the abnormal activation of internal pancreatin due to various causes, resulting in damage to the pancreatic acinar cells and the release of inflammatory factors, which, in turn, leads to a systemic inflammatory response $(16,17)$. Ulinastatin is a glycoprotein hydrolase inhibitor which can be extracted and refined from the fresh urine of healthy adult males. Ulinastatin is also known as a urinary trypsin inhibitor (UTI), possessing inhibitory activities towards proteolytic and lipolytic enzymes such as trypsin and elastase. Thus, UTIs have a treatment effect for AP. We found that ulinastatin treatment can reduce the 1-week mortality rate and APACHE-II score of SAP patients; the best results were seen in the 600,000 IU group. Although the course of SAP runs much longer than 1 week, ulinastatin can improve patient prognosis based on the above indicators. However, definitive confirmation requires further research.

From a clinical viewpoint, treatment with ulinastatin during the early stages of SAP can effectively improve abdominal pain and reduce the time to recover a normal heart and respiratory rate. Our study showed that different doses of ulinastatin had significantly different effects in reducing the abdominal pain relief time and the time to recover a normal respiratory rate, but not the time to recover a normal heart rate. This may be because abdominal pain and increased respiratory rate in patients with SAP are associated with elevated intra-abdominal pressure and acute lung injury during the early stages of the disease. Previous studies have shown that elevated intra-abdominal pressure and acute lung injuries are related to inflammation, which can be suppressed by ulinastatin $(18,19)$. To an extent, increasing the ulinastatin dose can reduce intra-abdominal pressure and fluid exudation in the lungs, thus resulting in the different dose responses to ulinastatin in terms of reducing abdominal pain relief time and the time taken to recover a normal respiratory rate, but not the time taken to recover a normal heart rate.

With regards to serological indicators, the ulinastatin treatment groups showed a marked reduction in blood glucose, CRP, and WBC levels compared to the control group. The 600,000 IU group showed a more noticeable reduction in WBC count compared to other treatment groups, but the difference in CRP levels was not significant. This finding is not consistent with other studies $(20,21)$ and may therefore require investigation through further clinical research. Moreover, PCT levels before and after 1 week of treatment did not show a significant difference in any of the treatment groups. It is known that PCT levels are selectively responsive to bacterial infections and are only mildly or not responsive to aseptic inflammation and viral infections. In patients with SAP, there is usually no significant infection present at 1 week after disease onset; thus, the above-mentioned finding would be expected. However, there is a discrepancy between the findings of this study and the expected clinical outcomes. This may be due 
to the limited sample size, the relatively small ulinastatin doses used, and the fact that this study focused on SAP and excluded the moderate and mild forms of this condition. In addition, this was a retrospective study, which means there was a risk of recall and selection bias. Patients were also subject to influences from a range of external factors, including non-medical factors, during their hospital stay. Therefore, this topic warrants further studies with a larger sample size and increased ulinastatin dose.

In conclusion, the treatment of patients with SAP with ulinastatin can reduce the 1-week mortality rate, decrease the APACHE-II score, improve clinical symptoms (time to abdominal pain relief and time to recover a normal heart and respiratory rate), and reduce serological marker levels (blood glucose, CRP, and WBC). Treatments with different doses of ulinastatin shows varying efficacy with regards to improvements in these outcomes. Therefore, an increase in ulinastatin dose within a certain range may increase the efficacy of SAP treatment but supporting evidence from further clinical research is still required.

\section{Acknowledgments}

Funding: None.

\section{Footnote}

Conflicts of Interest: All authors have completed the ICMJE uniform disclosure form (available at http://dx.doi. org/10.21037/apm.2020.04.19). The authors have no conflicts of interest to declare.

Ethical Statement: The authors are accountable for all aspects of the work in ensuring that questions related to the accuracy or integrity of any part of the work are appropriately investigated and resolved. The study was approved by the ethics committee of the First Affiliated Hospital, Anhui Medical University (No. QuickPJ2019-17-21). Informed consent was waived because the study was retrospective in design. The study was performed in accordance to the Helsinki Declaration. Individual patient data were anonymized and stored in an encrypted computer.

Open Access Statement: This is an Open Access article distributed in accordance with the Creative Commons Attribution-NonCommercial-NoDerivs 4.0 International License (CC BY-NC-ND 4.0), which permits the non- commercial replication and distribution of the article with the strict proviso that no changes or edits are made and the original work is properly cited (including links to both the formal publication through the relevant DOI and the license). See: https://creativecommons.org/licenses/by-nc-nd/4.0/.

\section{References}

1. Peery AF, Crockett SD, Murphy CC, et al. Burden and Cost of Gastrointestinal, Liver, and Pancreatic Diseases in the United States: Update 2018. Gastroenterology 2019;156:254-272.e11.

2. Fagenholz PJ, Fernandez-del Castillo C, Harris NS, et al. Direct medical costs of acute pancreatitis hospitalizations in the United States. Pancreas 2007;35:302-7.

3. Petrov MS, Yadav D. Global epidemiology and holistic prevention of pancreatitis. Nat Rev Gastroenterol Hepatol 2019;16:175-84.

4. Xiao AY, Tan ML, Wu LM, et al. Global incidence and mortality of pancreatic diseases: a systematic review, metaanalysis, and meta-regression of population-based cohort studies. Lancet Gastroenterol Hepatol 2016;1:45-55.

5. Banks PA, Bollen TL, Dervenis C, et al. Classification of acute pancreatitis--2012: revision of the Atlanta classification and definitions by international consensus. Gut 2013;62:102-11.

6. Mole DJ, Olabi B, Robinson V, et al. Incidence of individual organ dysfunction in fatal acute pancreatitis: analysis of 1024 death records. HPB (Oxford) 2009;11:166-70.

7. Chandrasekaran P, Gupta R, Shenvi S, et al. Prospective comparison of long term outcomes in patients with severe acute pancreatitis managed by operative and non operative measures. Pancreatology 2015;15:478-84.

8. Wang G, Liu Y, Zhou SF, et al. Effect of Somatostatin, Ulinastatin and Gabexate on the Treatment of Severe Acute Pancreatitis. Am J Med Sci 2016;351:506-12.

9. Lagoo JY, D'Souza MC, Kartha A, et al. Role of Ulinastatin, a trypsin inhibitor, in severe acute pancreatitis in critical care setting: A retrospective analysis. J Crit Care 2018;45:27-32.

10. Chen Q, Hu C, Liu Y, et al. Safety and tolerability of high-dose ulinastatin after 2-hour intravenous infusion in adult healthy Chinese volunteers: A randomized, doubleblind, placebo-controlled, ascending-dose study. PloS One 2017;12:e0177425.

11. Pan $\mathrm{Y}$, Fang $\mathrm{H}, \mathrm{Lu} \mathrm{F}$, et al. Ulinastatin ameliorates tissue damage of severe acute pancreatitis through modulating 
regulatory T cells. J Inflamm (Lond) 2017;14:7.

12. Xu Q, Yan Q, Chen S. Ulinastatin is effective in reducing mortality for critically ill patients with sepsis: a causal mediation analysis. Sci Rep 2018;8:14360.

13. Qian Y, Chen Y, Wang L, et al. Effects of baicalin on inflammatory reaction, oxidative stress and $\mathrm{PKDl}$ and $\mathrm{NF}-\mathrm{kB}$ protein expressions in rats with severe acute pancreatitis. Acta Cir Bras 2018;33:556-64.

14. Zhang Y, Yan L, Han W. Elevated Level of miR-551b-5p is Associated With Inflammation and Disease Progression in Patients With Severe Acute Pancreatitis. Ther Apher Dial 2018, 22:649-55.

15. Ye C, Wang R, Wang M, et al. Leptin alleviates intestinal mucosal barrier injury and inflammation in obese mice with acute pancreatitis. Int J Obes (Lond) 2018;42:1471-9.

16. Rotstein OD. Circulating cytokines in predicting development of severe acute pancreatitis. Crit Care 2014;18:575.

17. Sharma D, Jakkampudi A, Reddy R, et al. Association of Systemic Inflammatory and Anti-inflammatory

Cite this article as: $\mathrm{He} \mathrm{HW}$, Zhang $\mathrm{H}$. The efficacy of different doses of ulinastatin in the treatment of severe acute pancreatitis. Ann Palliat Med 2020;9(3):730-737. doi: 10.21037/ apm.2020.04.19
Responses with Adverse Outcomes in Acute Pancreatitis: Preliminary Results of an Ongoing Study. Dig Dis Sci 2017;62:3468-78.

18. Xu J, Cui Y, Tian X. Early Continuous Veno-Venous Hemofiltration Is Effective in Decreasing Intra-Abdominal Pressure and Serum Interleukin-8 Level in Severe Acute Pancreatitis Patients with Abdominal Compartment Syndrome. Blood Purif 2017;44:276-82.

19. Meng Y, Sha S, Yang J, et al. Effects of Tec Tyrosine Kinase Inhibition on the Inflammatory Response of Severe Acute Pancreatitis-Associated Acute Lung Injury in Mice. Dig Dis Sci 2019;64:2167-76.

20. Stirling AD, Moran NR, Kelly ME, et al. The predictive value of C-reactive protein (CRP) in acute pancreatitis - is interval change in CRP an additional indicator of severity? HPB (Oxford) 2017;19:874-80.

21. Y1lmaz EM, Kandemir A. Significance of red blood cell distribution width and C-reactive protein/albumin levels in predicting prognosis of acute pancreatitis. Ulus Travma Acil Cerrahi Derg 2018;24:528-31. 